\title{
Huddling and Independent Feeding of Neonatal Rats Can be Facilitated by a Conditioned Change in Behavioral State
}

\author{
REGINA M. SULLIVAN \\ City University of New York \\ Hunter College \\ New York, New York \\ STEPHAN C. BRAKE \\ MYRON A. HOFER \\ New York State Psychiatric Institute \\ at Columbia University \\ New York, New York \\ CHRISTINA L. WILLIAMS \\ Barnard College of Columbia University \\ New York, New York
}

\begin{abstract}
Infant rat pups were exposed to a novel odor (orange or cedar) while they received tactile stimulation (stroking of the body) or were presented with odor or stroking alone. The effects of these treatments were assessed on pups' performance in a huddling test (Experiment 1 and 2 ) and an independent feeding test (Experiment 3). During these tests, only pups that had received the simultaneous presentation of the odor and stroking exhibited an increase in huddling and feeding. The increase in these behaviors was dependent on the presence of the conditioned odor during testing.
\end{abstract}

Hall (1979) has shown that when food-deprived newborn rats ingest milk delivered through a tongue cannula or licked from the floor, their intake is accompanied by intense behavioral activation. This response is characterized by the onset of mouthing, probing, rolling, locomotion and head lifting. If food-deprived infants ingest milk while they are exposed to a novel odor, they will orient to and maintain contact with that odor in subsequent tests. In contrast, pups exposed to an odor without simultaneous milk ingestion and the accompanying behavioral response will continue to avoid the odor (Johanson \& Hall, 1982; Johanson \& Teicher, 1980).

Changes in behavioral state can also be elicited in neonates by other stimuli. These include: various tactile stimuli (e.g., stroking a pup's back or pinching its tail; Pedersen, Williams, \& Blass, 1982; Sullivan, Hofer, \& Brake, 1986; Sullivan \& Leon, 1986),

Reprint requsts should be sent to Regina M. Sullivan, Department of Psychobiology, University of California, Irvine, California 92717, U.S.A. 
electrical stimulation of the medial forebrain bundle (Moran, Lew, \& Blass, 1981), at least one pharmacological agent (e.g., amphetamine; Pedersen et al., 1982), and certain natural odors (e.g., maternal saliva; Sullivan et al., 1986). Like milk, these stimuli cause pups to show behavioral activation; stimulated pups increase their activity, and display a number of fragmented behaviors resembling feeding, suckling, and sexual responses. As illustrated in the previous article (Sullivan et al., 1986), pairing a novel odor with any one of these activating stimuli can also change pups' preference for that odor when it is next encountered (also see: Alberts \& May, 1984; Brake, 1981; Pedersen et al., 1982; Sullivan \& Hall, 1985).

When an odor is paired with a stimulus that causes these changes in behavioral state, it acquires more than just the ability to alter pups' preference for the odor. Specific conditioned appetitive responses (i.e., mouthing, probing, etc.) can be elicited by presentation of a novel odor which has previously been paired with intraoral infusions of milk (Johanson, Polefrone, \& Hall, 1984). These conditioned responses are dependent upon the milk-induced behavioral state (see Johanson \& Hall, 1982). As well, Pedersen et al. (1982) found that pups which are stimulated mechanically (by stroking) or pharmacologically (by amphetamine) in the presence of a novel odor, attach to the cleansed nipples of a dam only when that odor is present. These data suggest that a novel odor which has been paired with a stimulus which changes behavioral state acquires both the ability to cause pups to orient to and maintain contact with the odor, as well as the ability to facilitate certain appetitive behaviors (e.g., mouthing, probing, suckling).

These studies raise a number of questions regarding the nature of this type of learning in the neonate: (1) What determines a pup's behavioral response to an odor that has previously been paired with behavioral activation? Does the presence of the conditioned odor during testing facilitate certain prepotent or well-practiced behaviors that are often associated with odors in the nest, or is a nonspecific behavioral state being reactivated by the odor? (2) Is this change in behavior due to a type of associative learning, or are other nonassociative mechanisms (e.g., habituation, sensitization) more parsimoneous explanations. To address these questions, pups were exposed to a novel odor (orange extract or cedar odor) and a tactile stimulus that changes pups' behavioral state. One hour later, pups were reintroduced to the odor during two test situations. In Experiments 1 and 2 the odor was presented in a situation that allowed pups to huddle; in Experiment 3 the odor was presented while pups were allowed to feed independently of the dam. These behaviors were chosen because they represent neonatal precursors to two different adult motivational systems: behavioral thermoregulation and deprivation-dependent feeding.

\section{Experiment 1}

In the first experiment, the huddling behavior of pups in the presence of a novel odor was assessed after pups had had previous exposure to that novel odor and stroking. Huddling behavior was chosen because in the week-old rat it is activated by a homeostatic need; when the dam is absent pups regulate their body temperature through active huddling (Alberts \& Brunjes, 1978). Although pups can and do use olfactory cues to guide and facilitate other behaviors during the first week of life (e.g., suckling; Hofer, Shair, \& Singh, 1976; Teicher \& Blass, 1976, 1977) olfactory cues do not normally facilitate huddling until pups reach 15 days of age (Alberts \& Brunjes, 1978). Thus, we wondered whether pups' motivation to huddle could be enhanced by novel olfactory stimuli that had previously been paired with a change in behavioral state. 
A paradigm developed by Alberts (1978), for the systematic analysis of huddling was used. Pups were observed in a compartment which contained a single sacrificed littermate, warmed to $28^{\circ} \mathrm{C}$. Although this is not a typical situation in which huddling occurs, Alberts has shown that this controlled huddling test permits accurate and reliable observational measures and quantification (Alberts, 1978).

\section{Methods}

Subjects. The subjects were 24 male and 24 female Marland Farm Wister rats, 4-5 days of age, selected from 6 litters born at the animal care facilities of Montefiore Medical Center. One male and one female from each litter were assigned to each treatment condition. Pups were housed with their dam in a Plexiglas terraria $(40 \times 20 \times 24 \mathrm{~cm})$, on pine shavings, in a temperature $\left(22^{\circ} \mathrm{C}\right)$ and humidity-controlled room. Dams were allowed free access to food and water, and were maintained on a 12:12-hr light-dark cycle with light onset at 7:00 AM.

Deprivation conditions. Pups were separated from their dam 3-4 hrs before the start of treatment. During the deprivation period, pups were housed together in a warm $\left(31^{\circ} \mathrm{C}\right)$, moist incubator. Deprivation of this duration is within that which is normally experienced by a pup in the nest (Barnett, 1963).

Treatment procedure. Pups were randomly assigned to one of the following treatment conditions: (1) Orange \& Stroking, simultaneous presentation of orange odor and stroking for $10 \mathrm{~min}$; (2) Orange, presentation of the orange odor for $10 \mathrm{~min}$ with the pups left undisturbed; (3) Stroking, 10 min of stroking but with no added odor; and (4) Naive, 10 min with no stroking and no orange odor. During treatment, pups were placed in small tubs inside a warm $\left(31^{\circ} \mathrm{C}\right)$, moist incubator. The orange odor used during treatment was prepared by spraying $1.25 \mathrm{ml}$ of orange extract (Ann Page) onto $500 \mathrm{ml}$ of pine shavings. Several pieces of shavings were then suspended $5 \mathrm{~cm}$ above the treatment tub. In the no-odor conditions ( 2 and 4 ), no shavings were suspended above the test chamber. Pups were stroked continuously with a soft sable-hair brush, in a manner similar to the way a dam licks and manipulates her pups. Pups were stroked on the backs, legs, belly and anogenital region (see Pedersen et al., 1982, for a discussion of the activating properties of maternal licking).

Testing procedure. Pups were tested $1 \mathrm{hr}$ after treatment. The apparatus and testing conditions were similar to those used by Alberts (1978). Animals were tested at room temperature $\left(25^{\circ} \mathrm{C}\right)$ in a Plexiglas box $(12 \times 17 \times 4 \mathrm{~cm})$ with a $(.4 \times .4-\mathrm{cm}$ grid $)$ wire-mesh floor. A sacrificed littermate warmed to slightly above room temperature (approximately $28^{\circ} \mathrm{C}$ ) served as the huddling target. It was placed ventral side down against the long side of the test box. The orange odor was presented during the test by suspending several pine shavings sprayed with orange extract $10 \mathrm{~cm}$ above the center of the test box. At the start of the test, the subject was placed into the box with its nose $3 \mathrm{~cm}$ from the target pup.

Data collection. The test consisted of a 6 min observation period. A time sampling technique was used, so that the subject's behavior was observed every $20 \sec$ for 3-4 $\mathrm{sec}$, resulting in a total of 19 observations for each test pup. At each observation, it was recorded whether the subject was in contact with the target pup, and which of two specific behaviors was used by the pups to burrow into the target: (1) Pawing, paw in contact with the target pup; (2) Probing, nosing of the target pup. One-third of the litters were tested by an observer who was blind to the experimental conditions, two-thirds by an observer who knew the pup's treatment. 


\section{Results and Discussion}

As can be seen in Figure 1, the pups that received simultaneous presentation of the orange odor and stroking during treatment subsequently spent more time in contact (mean number of intervals pups were seen in contact) with the target pup than pups in each of the other treatment groups $(F(3,44)=19.25, p<.01$; post-hoc Tukey tests revealed that pups in the Orange \& Stroking group were in contact with the huddling target significantly more than pups in the other three groups, $p<.01$ ). These pups also engaged in active huddling behaviors more often than did pups in the other three groups. Specifically, they spent more time probing $(F(3,44)=4.37, p<.05)$ and pawing $(F(3,44)$ $=8.50, p<.01$ ) the target pup. Together, the data indicate that huddling behavior of 4- to 5-day old pups is increased by the presence of the orange odor, if the odor had previously been paired with tactile stimulation.

There are at least three possible explanations of this phenomenon: (1) Stroking pups in an orange-scented environment might produce a strong behavioral arousal state that was still present during testing. This arousal may have increased huddling, regardless of whether orange was present during the test. (2) Another possibility is that the orange odor acquired the capacity to increase huddling as the result of association with the behavioral state induced by stroking. During testing, the conditioned state may have increased pups' motivation to huddle. (3) A third possibility is that huddling was not actually increased in the pups that had been stroked in the presence of the odor. Instead, the seeming increase was caused by a disruption of huddling by the pups in the three control groups, caused by their aversion to the orange odor which scented the test chamber. It is possible that stroking pups in the presence of a novel odor simply eliminated pups' normal aversion to this odor, so that their huddling was not disrupted during the test. These possibilities were examined in Experiment 2.

\section{Experiment 2}

The treatment procedure of Experiment 1 was repeated, but half of the pups in each condition were tested in the presence of orange odor and half were tested in its absence. If the pups are activated by the presence of the orange odor during the test, then they should huddle more than pups tested in its absence. On the other hand, if pups are inhibited by the presence of the odor during the test, then they should huddle less than pups tested in its presence.

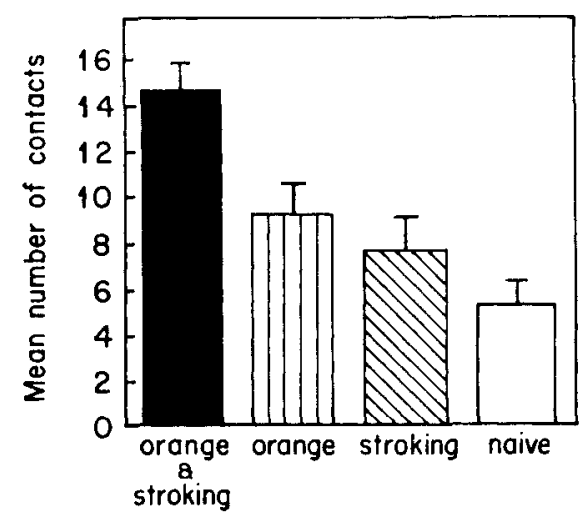

Fig. 1. Mean number of intervals in contact with a target pup in a huddling test. 


\section{Method}

Subjects. The subjects were 24 male and 24 female rat pups from six different litters born and raised under the same conditions described in Experiment 1. Three male and three female pups were assigned to each of the treatment-test condition. Only one pup from each litter was used in each experimental condition.

Procedure. The deprivation and treatment conditions were similar to those of Experiment 1 . The testing procedure was changed. Half of the pups in each treatment condition were tested with orange-scented wood shavings suspended above the test chamber, and half were tested with no orange odor present.

\section{Results and Discussion}

As can be seen in Figure 2, pups in the Orange \& Stroking group tested in the presence of the orange odor spent more time intervals in contact with the target pup than did pups in all other groups $(F(3,44)=7.99, p<.01)$. The results suggest that the differences in huddling behavior observed in Experiment 1 were due to the ability of orange odor to increase the number of intervals that pups in the Orange \& Stroking condition were in contact with the huddling target. In addition, it does not appear that the presence of the orange odor during the test had any aversive or disruptive effects on the huddling behavior. The number of sampling periods in which pups in the three control groups were in contact with the huddling target was about the same regardless of whether the pups were tested in the presence or absence of the orange odor. This study also provides evidence that the increased huddling of the Orange \& Stroking group is not due to arousal dictated during training, since the pups stroked in orange odor increased their huddling only when orange was present during testing. However, it is still possible that some arousal from treatment was still present during testing, and that this arousal combined with the arousal produced by the target pup increased huddling.

\section{Experiment 3}

In Experiment 2, the specificity of the response elicited by an odor previously paired with stroking was examined. In the present experiment, we tested whether another motivational state, hunger, could be facilitated by the presence of the odor. This behavioral

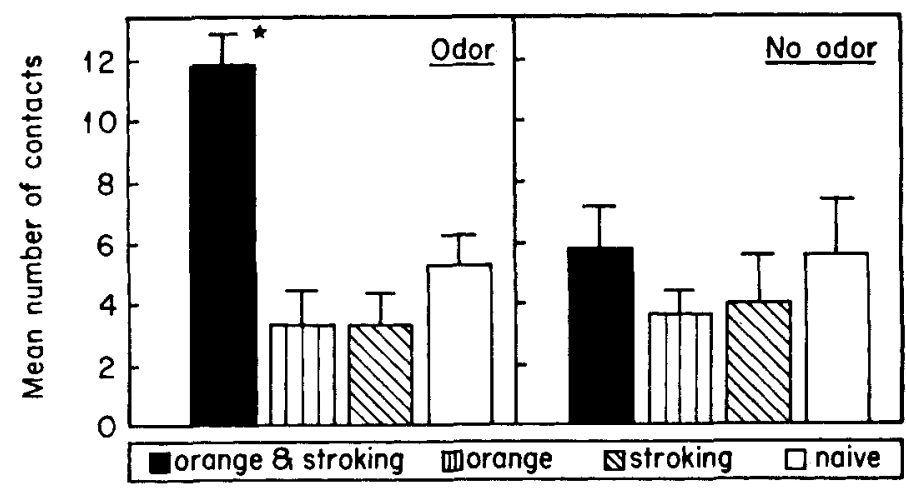

Fig. 2. Mean number of intervals in contact with a target pup during a huddling test. Pups were tested in the presence of the orange odor or in its absence. The star indicates that pups in this group spent significantly more intervals in contact with the huddling target than pups in the other groups. 
system was chosen for several reasons. First, there exists in the neonate an ingestive system that appears to be a simple version of the adult ingestive system and may be developmentally continuous with it (Hall \& Bryan, 1980). Thus, as huddling is a primitive form of behavioral thermoregulation, licking and lapping milk in a warm incubator may be a primitive form of adult deprivation-dependent ingestion. Second, demonstrations of conditioning based on behavioral activation usually employ behaviors that rat pups normally utilize in the nest (suckling behaviors, Pedersen et al., 1982; huddling, Experiment (1)), and which are often preceded by behavioral activation caused by maternal stimulation (i.e., licking, retrieving). Therefore, it is possible that stroking pups in the presence of a novel odor can only facilitate behaviors that are naturally preceded by activation (see Pedersen et al., 1982 for discussion of this hypothesis). The purpose of Experiment 2 was to investigate whether another motivational system in the neonate, one that is not utilized in the nest, can be facilitated by odor-stroking pairing.

In addition, we wished to examine whether the phenomenon was specific to orange odor or whether other scents could be utilized as novel odors. To do this, we presented cedar odor during treatment and testing. Feeding tests were conducted in the manner described by Hall and Bryan (1980). Pups kept in a warm, moist incubator were required to consume milk spread on the surface beneath them. Milk intake, probing, mouthing, and general activity were measured.

\section{Methods}

Subjects. The subjects were 40 male and 40 female pups, 5-6 days of age, selected from 10 litters of Charles River CD dams. Five males and five females were assigned to each condition, with no more than one pup from a litter in each condition. Litters were born and raised at the Duke University animal care facilities. Pups were housed with their dams on pine shavings in a temperature and humidity controlled room. Dams were allowed free access to food and water and were maintained on a 12:12-hr light-dark cycle with lights on at 7:00 AM.

Deprivation conditions. Pups were separated from their dam $18 \mathrm{hr}$ prior to the start of treatment. During the deprivation period, pups were housed together in a warm $\left(31^{\circ} \mathrm{C}\right)$ moist incubator. This long deprivation period was imposed in order to ensure that all pups consumed milk during the test period (see Hall \& Bryan, 1980).

Treatment. Pups were randomly assigned to one of the following treatment conditions: (1) Cedar \& Stroking, simultaneous exposure to cedar odor and stroking for 10 $\mathrm{min}$; (2) Cedar, exposure to cedar odor for $10 \mathrm{~min}$ without added stimulation; (3) Stroking, exposure to stroking for 10 min with no added odor; and (4) Naive, 10 min with no stroking and no added odor. During treatment, pups were placed in individual tubs in a warm $\left(31^{\circ} \mathrm{C}\right)$ incubator. Cedar odor was produced by blowing an airstream through a jar containing cedar extract. The airstream was then directed into the treatment chamber and was delivered just above the pup's snout. Stroking consisted of continuous tactile stimulation over all areas of the pups' bodies with a soft sable-hair brush.

Testing. A 12-min feeding test began $1 \mathrm{hr}$ following treatment. The procedure was based on that described by Hall and Bryan (1980). Body weights were taken immediately before and after the test and the percentages of body weight gain was used as the primary dependent variable. At the start of testing, pups were placed individually in clear plastic tubs $(5 \times 10 \mathrm{~cm})$ in a warm $\left(35^{\circ} \mathrm{C}\right)$ incubator. A paper towel soaked with $5 \mathrm{cc}$ of warm $\left(32^{\circ}\right.$ to $34^{\circ} \mathrm{C}$ at start of test) Half and Half $\left(\frac{1}{2}\right.$ milk, $\frac{1}{2}$ cream) was placed on the floor of the container. Half of the pups in each condition were exposed to cedar odor via a directed air stream for the duration of the test and half were exposed to air only. Every 
$30 \mathrm{sec}$ throughout the test, it was noted whether the pups were mouthing or probing, and general activity was rated using the method described by Hall and Bryan (1980): 0, not active; 1 , movement of part of the body, e.g., face washing, head raising; 2 , movement of the entire body, e.g., head and limb movements; 3, extreme behavioral activity, e.g., rolling over, wall climbing.

\section{Results and Discussion}

As can be seen in Figure 3, pups in the Cedar \& Stroking group that were tested in the presence of the cedar odor gained more weight during the test than did pups in all other groups (ANOVA yielded a test effect, $F(1,63)=8.779, p<.01$, and a treatment $\times$ test interaction, $F(3,63)=5.817, p<.01)$; the interaction reflected the fact that the presence of the odor during testing enhanced feeding. Post-hoc Tukey tests revealed that the Cedar \& Stroking group tested in the presence of the odor differed significantly from each of the other treatment-test conditions $(p<.05)$. These results indicate that although the presence of cedar odor during testing enhanced milk consumption, regardless of previous treatment, pups trained with Cedar and Stroking tested in the presence of cedar odor consumed more milk than pups in all other treatment-test conditions.

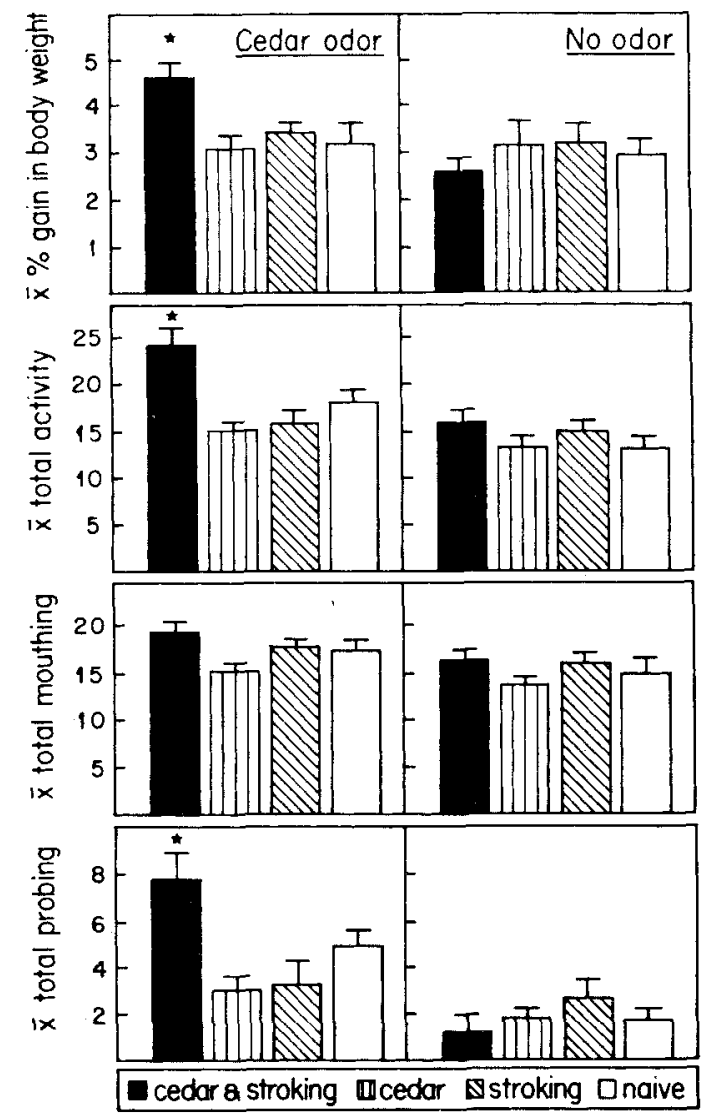

Fig. 3. Mean percentage of body weight gain, activity level, mouthing, and probing by pups during an independent feeding test. Pups were tested in the presence of cedar odor or in its absence. The stars indicate that pups in this group gained more weight, were more active, and engaged in more probing than pups in the other groups. 
Pups in the Cedar \& Stroking treatment condition tested in the presence of the odor were significantly more active than pups in all other treatment-test conditions (Fig. 3; ANOVA yielded a training effect, $F(3,63)=11.5, p<.01$; a test effect, $F(1,63)=$ $27.41, p<.01$; and a training $\times$ test interaction, $F(3,63)=4.45, p<.01)$. The interaction indicated that the presence of the odor during testing produced an enhancement in activity. Post-hoc Tukey tests revealed that the Cedar \& Stroking treatment group tested in the presence of the odor differed significantly from each of the other treatment-test conditions $(p<.05)$. The presence of the odor during testing enhanced activity levels but pups in the Cedar \& Stroking treatment condition tested in the presence of the odor had the highest activity level.

Pups in the Cedar \& Stroking treatment condition tested in the presence of the odor also exhibited increased probing. (Figure 3; ANOVA yielded a training effect, $F(3,63)$ $=3.39, p<.05$; a testing effect, $F(1,63)=34.81, p<.01$; and a treatment $\times$ test interaction, $F(3,63)=7.33, p<.01$.) The interaction indicated that the presence of the odor during testing produced an enhancement in probing. Post-hoc Tukey tests revealed that the mean of the Cedar \& Stroking treatment group tested in the presence of the odor differed significantly from each of the means of all other treatment-test conditions $(p<.05)$. Once again, the presence of the odor during testing enhanced probing but the pups in the Cedar \& Stroking training condition exhibited more probing than pups in any other treatment-test condition. The amount of mouthing exhibited by pups during testing did not differ among the various treatment-test conditions.

In summary, pups trained in the Cedar \& Stroking condition and tested in the presence of cedar odor consumed more milk, had higher activity levels, and probed more than pups in any other treatment-test conditions. These results suggest that stroking pups in the presence of an odor enables that odor to increase independent feeding behaviors and to increase food intake. This enhancement of feeding does not appear to be due to a long lasting behavioral arousal due to the combined stimulation of odor and stroking during training; Cedar \& Stroking pups tested without the presence of cedar odor did not differ from pups in other control groups. Moreover, the possibility that the presence of an odor during testing inhibits behavior seems unlikely; pups tested in the presence of the odor consumed more milk than pups tested without the odor. Therefore, Experiment 3 adds support to the hypothesis that an odor that has previously been paired with stroking acquires associations which allow it to enhance certain motivated behaviors. Since selffeeding is not a behavior that neonatal rats show in the nest, and is not a behavior that has ever been preceded by activation, it appears that the behaviors that are facilitated by conditioned odors are not necessarily behaviors utilized by the pup in the nest nor are they behaviors that have previously been associated with olfactory stimuli.

\section{General Discussion}

The present series of experiments illustrate that huddling and self-feeding of rat pups less than 1 week of age can be facilitated by the presence of a novel odor that has previously been paired with a behaviorally activating stimulus. These results complement previous findings. For example, when pups are placed into a two-choice test apparatus, they will orient to and maintain contact with an odor that had been paired with milkinduced or stroking-induced behavioral activation (Johanson \& Teicher, 1980; Sullivan \& Hall, 1985; Sullivan \& Leon, 1986; Sullivan et al., 1986). And, if pups are held with their snout on a nipple, an odor previously paired with stroking will elicit probing, mouthing, and nipple attachment (Pedersen et al., 1982). There are a number of possible ways that a novel odor can acquire properties which allow it to facilitate certain motivated 
behaviors. Some of these ideas have been addressed directly in the current studies, others have been explored in prior studies.

1. Pups exposed to an odor plus stroking may show increased behavior during the presentation of the test odor through some interaction or summation of arousing stimuli. In other words, the arousal produced by the novel odor and stroking may carry over to the test situation and be added to the arousing properties of the test odor. This combination may energize ongoing behavior. The results of Experiment 3 initially seem to give some support to this hypothesis since the presence of cedar odor during testing appeared to enhance feeding behaviors. However, when cedar was previously paired with stroking during training, cedar odor during testing had an even greater effect. Furthermore, in Experiment 2, the presence of orange odor during testing did not have arousing properties for control animals.

2. It is also possible that the augmentation of a response was not specific to the particular odor. Treatment may have sensitized the pup to any stimulus. This explanation was not tested directly in the current study, but has been examined in previous work using similar methodologies. Pedersen et al. (1982) showed that pups stroked in the presence of an orange odor did not attach to the washed nipples of their anesthetized dam if another novel odor (peppermint) was presented during the test. In this similar situation, sensitization, although possible, seems to be no more likely.

3. Pups could be responding differently because of some nonassociational process of adaptation/habituation. For example, the simultaneous presentation of an odor and an activating stimulus may alert the pup to the presence of the odor so that the odor is attended to more than it would otherwise. Control pups, which receive odor without stroking, may not attend as readily to the odor (they may even be asleep for some of the treatment period). Increased attention could lead to more rapid habituation to the odor's disruptive or inhibiting qualities. Thus, pups which are adapted to the odor might show more behavior during the test when the odor is present. However, the results of the current studies suggest that these processes do not appear to be responsible for the increased huddling and feeding seen in the simultaneous-exposure groups since the novel odors (orange or cedar) do not themselves inhibit huddling or feeding. In fact, first exposure to cedar odor tends to increase general activity and feeding in neonates (Experiment 3). And, while one could argue that simultaneous exposure to stroking and cedar simply amplifies the effects of odor exposure alone, one could not make such an argument for the effects of simultaneous exposure to stroking and orange since first exposure to orange does not appear to have arousing properties (Experiment 2).

4. We believe that the most likely explanation of the results of the present studies involves the establishment of an association between the novel odor and the behavioral state caused by the stroking. When the odor is presented during the test, pups that have been conditioned to the odor respond by showing enhanced or energized responses (i.e., more huddling or more feeding). This association may be similar to context conditioning that has been demonstrated in adult animals (see Balsam \& Tomie, 1985). For example, Balsam (1985) has shown that the general level of activity of ring doves can be conditioned to specific test contexts. In our studies, the behavioral state caused by stroking is associated with the new odor context. When the pup encounters this odor context during the test, the behavioral state caused by stroking is activated and the pups' ongoing behaviors are enhanced.

Regardless of the mechanisms by which pups' behavior is changed by the pairing of stroking and odor, these findings demonstrate that there is some plasticity to the behavioral responses that can be elicited by odors previously paired with activating stimuli. All of the behaviors that have been examined (activity and orientation, mouthing, 
probing, huddling and self-feeding) are ones that pups can and will display even in the absence of the test odor. Thus, the test odors appear to facilitate ongoing behavior, rather than to elicit new or different behaviors from the pups' repertoire. Perhaps the change in behavior induced by stroking can be viewed as the representation of an undifferentiated motivational state in the neonate. This idea is consistent with the findings of Moran, Schwartz, and Blass (1983) that hypothalamic electrical stimulation activates a variety of behavioral responses in pups less than 10 days of age (mouthing, probing, licking, lordosis-like responses, etc.). As development progresses these responses become organized into sequences and begin to take on the characteristics of specific motivated behaviors. In our studies, the test situation may help organize the undifferentiated responses of the pups into primitive forms of adult motivated behavior.

This interpretation of the phenomenon differs somewhat from that presented in a report by Pedersen et al. (1982) concerning how a novel odor can gain control over suckling behavior of neonates. They suggested that the phenomenon occurs because of higher order conditioning (as described by Rescorla, 1978) in the nest; arousal often precedes suckling, thus predicting the event. A novel odor which has been paired with a behaviorally activating event (like stroking) then takes on the predictive, as well as the arousing properties of activation. The current findings, that conditioned odors can also facilitate huddling and independent feeding behavior, suggest that this interpretation may not be valid in all circumstances. It would be difficult to see how an odor could come to predict independent feeding through higher order conditioning since the test situation is the first time the neonate has ever had the opportunity to mouth and lick milk from the floor. Therefore, we suggest an alternative mechanism. Only the behavioral state of arousal is conditioned, and not its predictive properties, and this state, in the presence of certain familiar contextual cues, causes the facilitation of ongoing behaviors.

Rosenblatt $(1979,1983)$ has proposed a similar framework for the development of olfactory-based social behaviors. He suggests that certain stimuli produce a non-specific arousal and that this arousal may be sufficient to produce an association between the arousing stimulus and a novel odor, resulting in the odor acquiring incentive. Once the odor has acquired incentive, it becomes capable of eliciting orientation to the source of the odor and a general behavioral arousal. Our results suggest that this conditioned arousal has the capacity to enhance a variety of ongoing behaviors.

\section{Notes}

Supported by NIMH Grant \# MH38465 awarded to Stephen Brake, NIMH Grant \# MH40430 to Myron Hofer and NIH Grant \# NS20671 to Christina L. Williams. The majority of this work was performed at Montefiore Medical Center, Albert Einstein College of Medicine, Bronx, NY, and at Barnard College, New York, NY. Part of the data was collected while the first author was a post-doctoral fellow at Duke University, in W.G. Hall's laboratory, and we thank him for the use of his lab and his advice. We also wish to thank Marcia Levin-Pelchat, Gordon Barr, Gerald Turkewitz, Peter Balsam and Warren Meck for advice and comments on drafts of this paper, and Susan Casselman for her assistance in data collection.

\section{References}

Alberts, J. R. (1978). Huddling by rat pups: multisensory control of contact behavior. J. Comp. Physiol. Psychol., 192:220-230.

Alberts, J. R., and Brunjes, P. C. (1978). Ontogeny of thermal and olfactory determinants of huddling in the rat. J. Comp. Physiol. Psychol., 92:897-906.

Alberts, J. R., and May, B. (1984). Nonnutritive thermotactile induction of filial huddling in rat pups. Dev. Psychobiol., 17:161-181. 
Balsam, P. D. (1985). The functions of context in learning and performance. In P. D. Balsam and A. Tomie (eds.), Context and Learning. Hillsdale, NJ: Erlbaum.

Balsam, P. D., and Tomie, A. (eds.) (1985). Context and Learning. Hillsdale, NJ: Erlbaum.

Barnett, S. A. (1963). A Study in Behavior. London: Methuen.

Brake, S. C. (1981). Suckling infant rats learn a preference for a novel olfactory stimulus paired with milk delivery. Science, 211:506-508.

Hall, W. G. (1979). Feeding and behavioral activation in infant rats. Science, 205:206-209.

Hall, W. G., and Bryan, T. E. (1980). The ontogeny of feeding in infant rats. II. Independent ingestive behavior. J. Comp. Physiol. Psychol., 94:746-756.

Hofer, M. A., Shair, H., and Singh, P. (1976). Evidence that ventral skin substances promote suckling in infant rats. Physiol. Behav., 17:131-136.

Johanson, I. B., and Hall, W. G. (1982). Appetitive conditioning in neonatal rats: conditioned orientation to a novel odor. Dev. Psychobiol., 15:379-397.

Johanson, I. B., Polefrone, J. M., and Hall, W. G. (1984). Appetitive conditioning in neonatal rats: conditioned ingestive responding to stimuli paired with oral infusion of milk. Dev. Psychobiol., 17:357-381.

Johanson, 1. B., and Teicher, M. (1980). Classical conditioning of an odor preference in 3-day-old rats. Behav. Neural Biol., 29: 132-136.

Moran, T. H., Lew, M. F., and Blass, E. M. (1981). Intracranial self-stimulation in 3-day-old rat pups. Science, 214:1366-1368.

Moran, T. H., Schwartz, G. J., and Blass, E. M. (1983). Organized behavioral responses to lateral hypothalamic electrical stimulation in infant rats. J. Neurosci., 3:10-19.

Pedersen, P. E., Williams, C. L., and Blass, E. M. (1982). Activation and odor conditioning of suckling behavior in 3-day-old rats. J. Exp. Psychol. Anim. Behav. Proc., 8:329-341.

Rescorla, R. A. (1978). Some implications of a cognitive perspective on Pavlovian conditioning. In S. H. Hulse, H. Fowler, and W. K. Honig (eds.), Cognitive Processes in Animal Behavior. Hillsdale, N.J.: Erlbaum.

Rosenblatt, J. S. (1979). The sensorimotor and motivational bases of early behavioral development of selected altricial mammals. In N. E. Spear and B. A. Campbell (eds.), Ontogeny of Learning and Memory. New York: Wiley. Pp. 1-38.

Rosenblatt, J. R. (1983). Olfaction mediates developmental transition in the altricial newborn of selected species of mammals. Dev. Psychobiol., 16:347-375.

Sullivan, R. M., and Hall, W. G. (1985). Classical conditioning of behavioral activation using intra-oral infusions of milk or stroking as reward in infant rats. Paper presented at the Annual Meeting of the International Society for Developmental Psychobiology, Dallas, Texas.

Sullivan, R. M., Hofer, M. A., and Brake, S. C. (1986) Olfactory-guided orientation in neonatal rats is enhanced by a conditioned change in behavioral state. Dev. Psychobiol, 19:615-623.

Sullivan, R. M. and Leon, M. (1986). Early olfactory learning induces an enhanced olfactory bulb response in young rats. Dev. Brain Res., 27:278-282.

Teicher, M. H., and Blass, E. M. (1976). Suckling in newborn rats: Elimination by nipple lavage, reinstated by pup saliva. Science, 193:422-425.

Teicher, M. H., and Blass, E. M. (1977). First suckling response of the new born albino rat: The role of olfaction and amniotic fluid. Science, 198: 635-636. 